\title{
The content of mucin MUC-2, -3 and -4 antigens in the bronchial mucosa membrane of chronic obstructive pulmonary disease patients during acute exacerbation - initial report
}

The authors declare no financial disclosure

\begin{abstract}
Introduction: Changes in mucin production and dyscrinia are common features of inflammation in chronic obstructive pulmonary disease (COPD). Immunohistochemical assessment of MUC-2, MUC-3, MUC-4 expression in the integumentary epithelium, goblet cells, the epithelium of mucous glands and stroma fusiform cells of the bronchial mucosa of COPD patients during an infectious and noninfectious exacerbation was performed.

Material and methods: 30 patients with stage III COPD were enrolled to the study. Patients were divided into 2 groups: group A -14 patients with non-infectious acute exacerbation of COPD (AECOPD) and group $B-16$ patients with infectious AECOPD. Fiberoptic bronchoscopy (FBS) and in vivo bronchial biopsy of bronchial mucosa were implemented to determine the extent and nature of bronchial inflammation. The optical density of specific color in bronchial structures was assessed using immunohistochemical staining to MUC-2, -3 and -4 antigens by means of primary monoclonal antibodies to these proteins, and visualization system Dako EnVision + System, Peroxidase (AEC). Results and conclusions: We detected that in different types of bronchial mucosa epithelial cells, during acute infectious exacerbation, a decrease of antigens MUC-2 and MUC-3 expression of a various degree may occur. This phenomenon in the stroma fusiform cells in AECOPD may be a sign of epithelial-mesenchymal transition, that may play a role in the development of an inflammatory process and progression of fibrosis in COPD.
\end{abstract}

Key words: mucins, bronchial mucosa, inflammation

Adv Respir Med 2017; 85: 3-7

\section{Introduction}

There is an evidence that mediators of inflammation may activate mucin gene regulation and remodulation of the respiratory tract (RT), including cell hyperplasia [1, 2]. Changes in mucin production and dyscrinia are common features of inflammation in chronic obstructive pulmonary disease (COPD), what logically justifies the importance of research on mucins influence in the progression of inflammatory processes in RT [3].

The aim of the study was an assessment of mucins MUC-2, -3 , and -4 as markers of inflammation in bronchial mucosa in COPD.

\section{Material and methods}

Fiberoptic bronchoscopy (FBS) and in vivo bronchial biopsy of bronchial mucosa were implemented to determine the extent and nature of bronchial inflammation in 30 patients with COPD.

The fresh material was kept for 22 hours in neutral buffered aqueous solution of $10 \%$ formalin. The specimens were dehydrated in increasing ethanol solutions, and fixed in paraffin. Five microns thick - sections were made for further assessment. Paraffin sections were mounted onto non-immunogenic glass slides (SuperFrost ${ }^{\circledR}$ Plus, 
Germany). The optical density (in conventional units) of specific color in bronchial structures were assessed using immunohistochemical staining to antigens MUC-2, MUC-3 and MUC- 4 by means of primary monoclonal antibodies to these proteins, and visualization system Dako EnVision + System, Peroxidase (AEC).

The color intensity (optical density) was objectively evaluated in the digital copies of micro-preparations optical images, using computer graphics microdencimetry (GIMP software, version 2.82 , license GPL) by probe method gradations in color intensity gradations (from 0 to 255) with logarithmic recalculation in conventional units of optical density (con.un.opt. density) from " 0 " (absolute transparency) to " 1 " (absolute opacity).

Patients included to the study suffered from stage III COPD and were divided into 2 groups: group A -14 patients with clinical signs of exacerbation but without signs of infection (non-infectious AECOPD) and group B - 16 patients with infectious exacerbation (infectious AECOPD) [1]. In the group $A$, there were 6 female patients (42.8\%), 3 active smokers (29.5 $\pm 2,4$ pack-years) and 11 ex-smokers. The average $\mathrm{FEV}_{1}$ for the group A was $38.9 \pm 4.3 \%$ of the predicted value. In group $B$, average age was $65.4 \pm 4.3$ years. There were $7(43.7 \%)$ female patients in this group. The mean duration of disease was 18.3 \pm 1.7 years. There were 5 active smokers and 15 ex-smokers. The average value of $\mathrm{FEV}_{1}$ for group B was $37.6 \pm 2.7 \%$ of predicted value. All patients signed an informed consent before entering the study. Institutional ethical commission approved the protocol.

\section{Results}

The positive immunohistochemical staining for MUC-2 antigen was observed in a few cells that were desquamated in the bronchial lumen (Fig. 1). The optical density was $0.128 \pm 0.010$ con.un.opt. density. Exactly the same quantities were obtained for surface bronchial epithelium (Table 1).

The expression of MUC-3 antigen was observed in the bronchial surface epithelium from moderate to weak magnitude with average numbers of color optical density of $0.201 \pm 0.021$ con.un.opt.density. The maximum expression of MUC-3 antigen in the bronchial surface epithelium is shown in Figure 3.

In goblet cells, the MUC-3 antigen expression has not been detected. In mucous glands, MUC-3

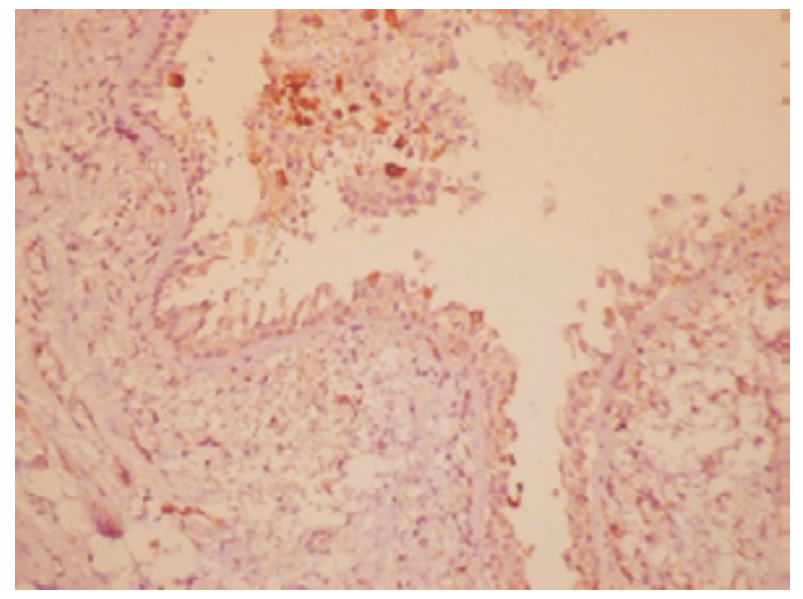

Figure 1. Group A - expression of MUC-2 antigen in desquamated bronchial epithelium - the immunohistochemical methods of visualization with diaminebenzidine. $0 \mathrm{~b} .20^{\times} .0 \mathrm{k} .10^{\times}$

Table 1. The optical density (in conventional units) of specific immunohistochemical staining of MUC-2, MUC-3, MUC-4 antigens expression in bronchial structures of patients with $\operatorname{COPD}(X \pm s x)$

\begin{tabular}{lccc}
\hline Immunohistochemical methods/localization & $\begin{array}{c}\text { Group A } \\
(\mathbf{n}=\mathbf{1 4})\end{array}$ & $\begin{array}{c}\text { Group B } \\
(\mathbf{n}=\mathbf{1 6})\end{array}$ & $\begin{array}{c}\text { Probability of differences (P) } \\
\text { in the average trend by Mann- } \\
\text {-Whitney method }\end{array}$ \\
\hline MUC-2/surface epithelium & $0,128 \pm 0,010$ & Not defined & p not calculated \\
MUC-2/goblet cells & $0,247 \pm 0,016$ & $0,196 \pm 0,015$ & $\mathrm{p}<0,05$ \\
MUC-2 /epithelium of mucous gland & $0,212 \pm 0,018$ & $0,103 \pm 0,008$ & $\mathrm{p}<0,01$ \\
MUC-2/MUC-2- positive spindle cells of stroma & $0,208 \pm 0,025$ & $0,206 \pm 0,028$ & $\mathrm{p}>0,05$ \\
MUC-3/surface epithelium & $0,201 \pm 0,021$ & $0,049 \pm 0,006$ & $\mathrm{p}<0,001$ \\
MUC-3/goblet cells & Not defined & Not defined & $\mathrm{p}$ not calculated \\
MUC-3/epithelium of mucous gland & $0,192 \pm 0,009$ & $0,065 \pm 0,007$ & $\mathrm{p}<0,001$ \\
MUC-3/MUC-3- positive spindle cells of stroma & $0,286 \pm 0,024$ & $0,289 \pm 0,028$ & $\mathrm{p}>0,05$ \\
MUC-4/any structure & Not defined & Not defined & $\mathrm{p}$ not calculated \\
\hline
\end{tabular}




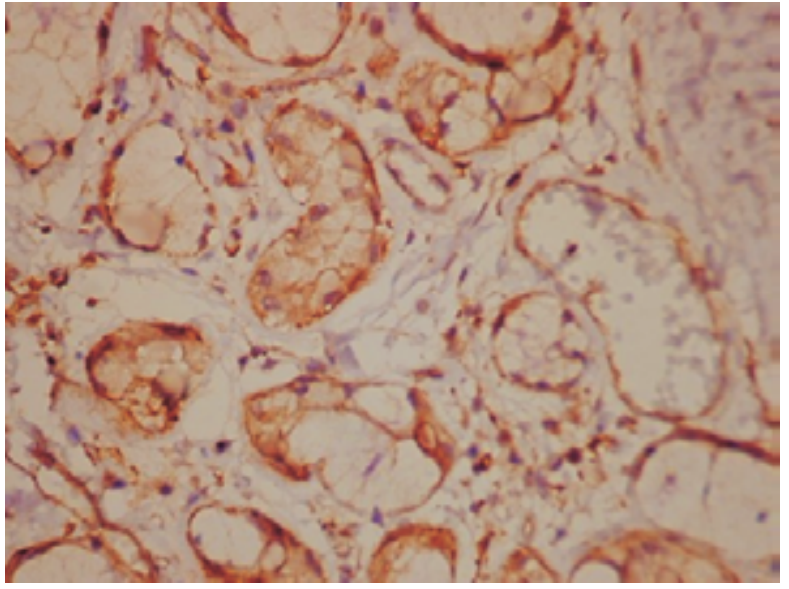

Figure 2. Group A - expression of MUC-2 antigen in the bronchial mucous glands epithelium and in spindle (fibroblast) cells of the bronchial stroma - immunohistochemical method and visualization with diaminebenzidine. $0 \mathrm{~b} \cdot 20^{\times} \cdot 0 \mathrm{k} \cdot 10^{\times}$

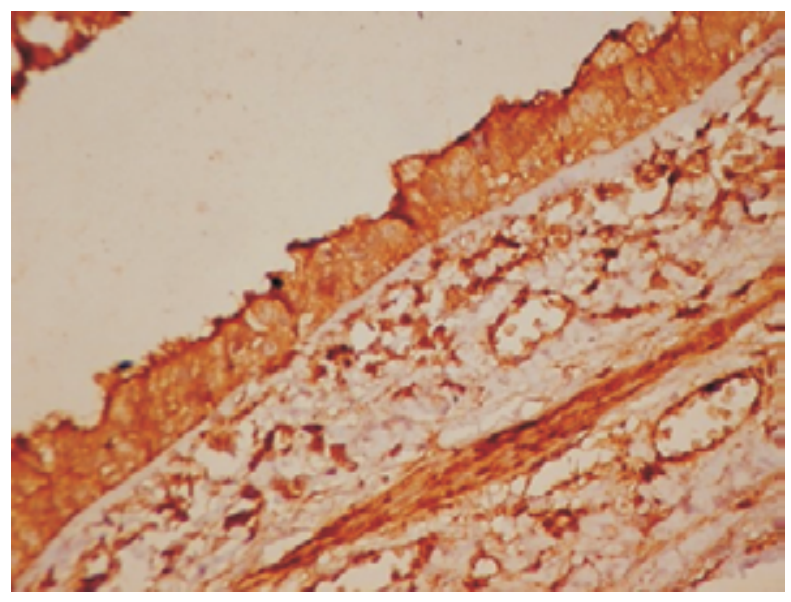

Figure 3. Group A - expression of MUC-3 antigen in the bronchial surface epithelium and spindle (fibroblast) cells of the bronchial stroma — the immunohistochemical method of visualization with diaminebenzidine. 0 b. $20^{\times} .0 \mathrm{k} .10^{\times}$

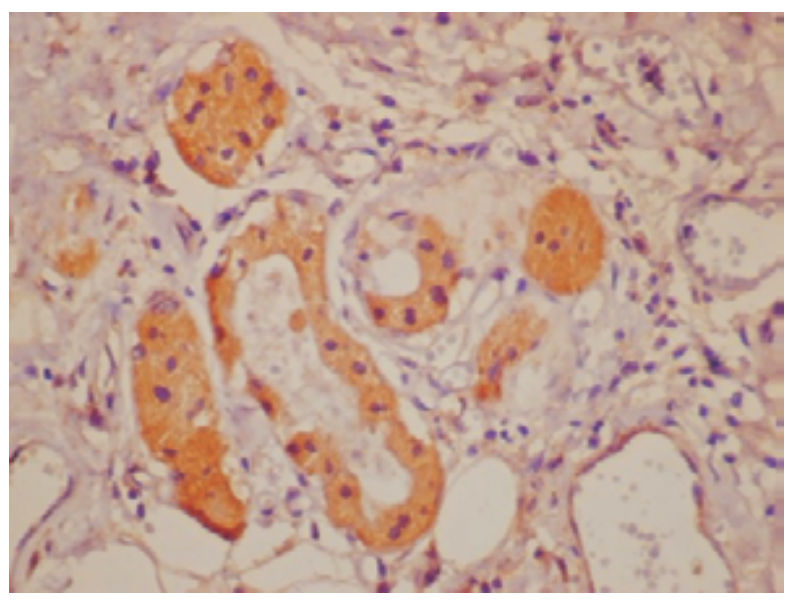

Figure 4. Group A - expression of MUC-3 antigen in bronchial mucous glands - the immunohistochemical method of visualization with diaminebenzidine. 0 b. $20^{\times} .0 \mathrm{k} .10^{\times}$ expression was absent in the majority of cells, and in those where it existed (Fig. 4), it could be assessed as moderate with average numbers of color optical density equal to $0.192 \pm 0.009$ con. un.opt.density.

In bronchial stroma, expression of MUC3 was determined in the spindle cells under sub-epithelial basal membrane (Fig. 3) with the moderate to high expression, with average magnitudes of color optical density of $0.286 \pm 0.024$ con.un.opt.density.

We observed a decrease by $75.7 \%$ in the expression of MUC-3 in bronchial epithelium in patients with infectious AECOPD comparing to non- infectious one, while in the epithelium of mucous glands - by $66.1 \%$. In spindle cells, MUC-3 expression did not depend on the nature of COPD exacerbation $-0.286 \pm 0.024$ and 0.289 $\pm 0.028(\mathrm{p}>0.05)$, respectively. In goblet cells, MUC-3 expression was not observed in both groups of patients.

\section{Discussion}

It has been found that during infectious AECOPD, in different types of epithelial cells of mucous membrane of the bronchial tree, a significant decline of MUC-2 and -3 antigens expression occurs.

The declined expression of MUC- 2 and -3 antigens in the fusiform cells of the stroma (fibroblasts) during a COPD exacerbation may confirm an epithelial-mesenchymal transition. Change of transformed epithelial cells is a reaction to various molecular factors which play a certain role in the development of an inflammatory process and progression of fibrosis in COPD.

In the AECOPD, an expression of secretorial MUC-2 and MUC-3 mucins, which contribute to the formation of mucus gel component was observed. Synthesis of MUC-2 and MUC-3 decreases in infectious exacerbation of stage III COPD compared to their expression in the non-infectious exacerbation in all structures except stroma spindle cells - fibroblasts. We believe that reduction of mucins expression in infectious AECOPD is mostly pronounced in the surface epithelium, the epithelium of the mucous glands and less in goblet cells. It can be explained by the influence of pathogenic microorganisms, which are present in a large amount in the mucous membranes and bronchial surface epithelium in infectious AECOPD, worsening the composition of normal mucus and mucociliary clearance [3]. Uniform expression of MUC-2 and -3, which appears in 
fibroblasts stroma, and does not depend on the nature of COPD exacerbation, is hypothetically related to epithelial-mesenchymal transition (EMT) phenomenon, when normal epithelial cells (e.g., mucosal glands) acquire the character of miofibroblast cells under the influence of certain factors, e.g., TGF- $\beta 1$ [4]. In EMT, epithelial cells which lost intercellular connections, move to the interstitium, where after getting full mesenchymal phenotype are involved into the synthesis of fibrous matrix [5].

As for MUC-4, it should be noted that in our study the expression of this antigen usually has not been determined in any structure. Only two patients had the expression of MUC-4 antigen in the bronchial surface epithelium, and it was hardly noticeable.

In group B, MUC-2 antigen expression in the surface epithelium is practically not-detectable. Thus, during infectious AECOPD, the protein production in the surface epithelium seems to be impaired. The decreased color intensity of MUC2 antigen in the goblet cells and mucous glands epithelium are presented in Table 1. Expression of MUC-3 antigen in the surface epithelium and that of the mucous glands was also decreased (Table 1). In goblet cells in infectious, as well as in non-infectious AECOPD, expression of MUC-3 antigen has not been detected.

Unlike the aforementioned epithelial cells, the decrease of expression of MUC-2 and MUC-3 antigens in spindle cells of bronchial stroma in infectious AECOPD has not been found. Thus, the formation of these molecules in cells that were likely undergone EMT did not change at infectious compared to non-infectious AECOPD. It may be an evidence, that transformed epithelial cells respond differently to molecular factors that influence the development of inflammation and progression of fibrosis in COPD than normal epithelial cells.

When the specific staining of the bronchial structures for MUC-2, MUC-3, MUC-4 antigens was assessed, the expression of various degree in surface bronchial epithelium and epithelium of the mucous glands, in goblet cells, stroma spindle cells (fibroblasts) in infectious and non -infectious AECOPD was detected. Therefore, in the surface of bronchial epithelium, expression of MUC-2 was detected in patients without evidence of infectious AECOPD. In the contrary, such expression was not observed in infectious AECOPD. MUC-2 expression in goblet cells was the highest, however it was $31 \%$ lower in infectious AECOPD in comparison to non-infectious one.
MUC-2 expression in the epithelium of mucous glands in patients with infectious AECOPD was twice less frequent in comparison to patients without features of infectious exacerbation. MUC2 expression in stromal spindle cells (fibroblasts) was observed on the same level of expression in the group A and B.

Expression of MUC-4 in bronchial surface epithelium was observed only in two patients with AECOPD from the group A. This was probably due to the low sensitivity threshold of the method to MUC4 antigen and its weak secretion in COPD. The membrane-bound MUC4 presented in the apical surface of ciliated cells, probably undergoes desquamation together with the epithelium and does not get into the sight of the investigated material.

Although the ultimate role of mucins is unknown, they can act as receptors or receptor ligands and are able to activate intracellular signaling cascades that affect the functions of the epithelial cells, also promoting the development of EMT [6, 7].

\section{Conclusions}

Changes in mucins production in the bronchial wall may be the sign of inflammation in COPD. During infectious AECOPD, in different types of epithelial cells of the bronchial mucosa, the expression of MUC-2 and MUC-3 antigens decreases. Immunohistochemical study of antigens MUC-2 and MUC-3 in the stroma spindle cells justifies the hypothesis on occurrence of EMT in bronchial mucosa in AECOPD.

Study of the functional role of mucins at the molecular level is essential for further research that will affect the prevention of the progression of COPD and its treatment.

\section{Conflict of interest}

The authors declare no conflict of interest.

\section{References:}

1. Global Initiative for Chronic Obstructive Lung Disease strategy for the diagnosis, management and prevention of chronic obstructive pulmonary disease: An Asia-Pacific perspective. Respirology. 2005; 10(1): 9-17, doi: 10.1111/j. 1440-1843.2005.00692.x.

2. Hackett TL, Warner SM, Stefanowicz D, et al. Induction of epithelial-mesenchymal transition in primary airway epithelial cells from patients with asthma by transforming growth factor -beta1. Am J Respir Crit Care Med. 2009; 180(2): 122-133, doi: 10.1164/rccm.200811-17300C, indexed in Pubmed: 19406982.

3. Linden SK, Sutton P, Karlsson NG, et al. Mucins in the mucosal barrier to infection. Mucosal Immunol. 2008; 1(3): 183-197, doi: 10.1038/mi.2008.5, indexed in Pubmed: 19079178. 
4. Mitchell E, Houles C, Sudakevitz D, et al. Structural basis for oligosaccharide-mediated adhesion of Pseudomonas aeruginosa in the lungs of cystic fibrosis patients. Nat Struct Biol. 2002; 9(12): 918-921, doi: 10.1038/nsb865, indexed in Pubmed: 12415289.

5. Nowrin K, Sohal SS, Peterson G, et al. Epithelial-mesenchymal transition as a fundamental underlying pathogenic process in COPD airways: fibrosis, remodeling and cancer. Expert Rev Respir Med. 2014; 8(5): 547-559, doi:
10.1586/17476348.2014.948853, indexed in Pubmed: 25113142.

6. Singh PK, Hollingsworth MA. Cell surface-associated mucins in signal transduction. Trends Cell Biol. 2006; 16(9): 467-476, doi: 10.1016/j.tcb.2006.07.006, indexed in Pubmed: 16904320 .

7. Sohal SS. Endothelial to mesenchymal transition (EndMT): an active process in Chronic Obstructive Pulmonary Disease (COPD)? Respir Res. 2016; 17: 20, doi: 10.1186/s12931-0160337-4, indexed in Pubmed: 26898357. 